\title{
COMPARISON OF THE PERFORMANCE OF DIFFERENT INSTRUMENTS IN THE STRAY NEUTRON FIELD AROUND THE CERN PROTON SYNCHROTRON
}

\author{
Eleni Aza ${ }^{1,2}$, Marco Caresana ${ }^{3}$, Christopher Cassell $^{3,4}$, Valeria Colombo ${ }^{3}$, Sanja Damjanovic ${ }^{1}$, \\ Simone Gilardoni ${ }^{1}$, Giacomo Paolo Manessi ${ }^{1,5, *}$, Michel Pangallo ${ }^{1}$, Daniel Perrin ${ }^{1}$ and Marco Silari ${ }^{1}$ \\ ${ }^{1}$ CERN, CH-1211 Geneva 23, Switzerland \\ ${ }^{2}$ Department of Physics, Aristotle University of Thessaloniki, GR-54124 Thessaloniki, Greece \\ ${ }^{3}$ Department of Energy, Politecnico of Milan, Via Ponzio 34/3, 20133 Milan, Italy \\ ${ }^{4}$ Centre for Medical Radiation Physics, University of Wollongong, NSW 2522, Australia \\ ${ }^{5}$ Department of Physics, University of Liverpool, L69 7ZE Liverpool, UK \\ *Corresponding author: giacomo.manessi@cern.ch
}

\begin{abstract}
This paper discusses an intercomparison campaign carried out in several locations around the CERN Proton Synchrotron. The locations were selected in order to perform the measurements in different stray field conditions. Various neutron detectors were employed: ionisation chambers, conventional and extended range rem counters, both commercial and prototype ones, including a novel instrument called LUPIN, specifically conceived to work in pulsed fields. The attention was focused on the potential differences in the instrument readings due to dead-time losses that are expected to affect most commercial units. The results show that the ionisation chambers and LUPIN agree well with the expected $H^{*}(10)$ values, as derived from FLUKA simulations, showing no relevant underestimations even in strongly pulsed fields. On the contrary, the dead-time losses of the other rem counters induced an underestimation in pulsed fields that was more important for instruments characterised by a higher dead time.
\end{abstract}

\section{INTRODUCTION}

A series of measurements with active neutron detectors was performed in selected locations around the CERN PS in 2011 and 2012. The instruments employed in the campaign, both commercial units and prototypes, are used for routine measurements at CERN or employed in the Radiation Monitoring System for Environment and Safety (RAMSES) ${ }^{(1)}$. The attention was focused on the potential differences in the instrument readings due to dead-time losses that are expected to affect most of the commercial units in pulsed neutron fields. The measuring locations were selected on the basis of the expected time structure of the losses in order to carry out the measurements in different stray field conditions.

During the measurement campaign the proton beams used for the fixed target physics at the Super Proton Synchrotron were extracted from the Proton Synchrotron (PS) at $14 \mathrm{GeV}$ using the continuous transfer technique. In the extraction phase, comparatively large losses are observed all around the PS. These losses are due to particles scattered by the electrostatic septum used to slice the beam. Figure 1 shows a scheme of the PS complex, which is composed of 100 combined-function magnets arranged in a lattice and interleaved by 100 straight sections.

The aim of the measurements was to evaluate the response of the instrumentation in three locations where the time structure of the losses is remarkably different and then to systematically intercompare their performances in a position where the stray field is extremely pulsed and intense, i.e. where pile-up effects and dead-time losses are expected.

\section{INSTRUMENTATION}

The instruments employed were the following: two extended range rem counters (LINUS $^{(2)}$ and Thermo FHT 762 Wendi-2), an extended range prototype detector called LUPIN ${ }^{(3)}$, available in two versions $\left({ }^{3} \mathrm{He}\right.$ and $\mathrm{BF}_{3}$ ), specifically conceived for applications in pulsed neutron fields, three commercial rem counters (Studsvik 2202D, Berthold LB6411 and Thermo FHT 751 BIOREM) and two customised Centronic IG5 ionisation chambers [pressurised Argon (A20) and Hydrogen type (H20)]. A detailed description of the detectors as well as of their response functions can be found in refs $(4,5)$.

\section{MEASUREMENTS}

The measurements were carried out in the following locations (Figure 1), where, from Monte Carlo simulations, it is known that the stray radiation field is dominated by neutrons: 


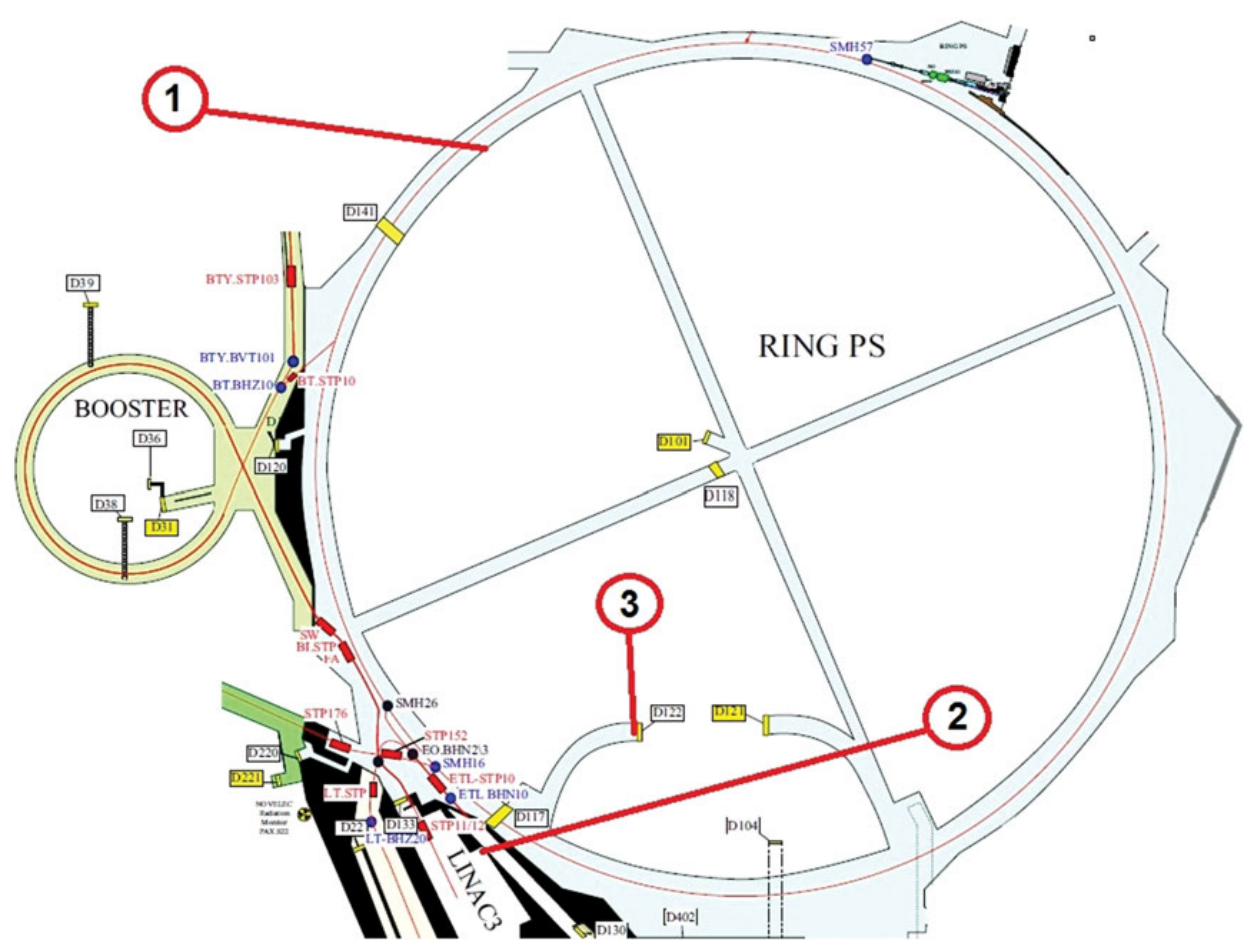

Figure 1. The PS accelerator complex. The red marks show the measuring locations discussed in the text.

(1) On Route Goward, close to SS42 (beam injection). Typical duration of the losses: $200-300 \mu \mathrm{s}$, smoothed by the ground shield (the street level is $6.4 \mathrm{~m}$ above the beam axis). Fraction of lost beam: $1-5 \%$. In SS42 the $1.4-\mathrm{GeV}$ proton beam from the PS Booster is injected in the PS.

(2) Inside the LINAC 3 building, close to SS16 (beam extraction). Typical duration of the losses: $2.1 \mu \mathrm{s}$. Fraction of lost beam: maximum $1 \%$.

(3) At the beginning of the access tunnel to SS16 (beam extraction). Typical duration of the losses: $2.1 \mu \mathrm{s}$. Fraction of lost beam: $1 \%$. The area is located at the accelerator level, just before an interlocked door.

In addition to the losses typical of each measuring position, one has to consider that extraction losses are distributed on the overall PS and are always to be added up. These losses are due to the slicing process of the beam, which is extracted from the PS in five turns. Each of these turn lasts for $2.2 \mu \mathrm{s}$ and the extraction losses have therefore a typical length of $\sim 11 \mu \mathrm{s}^{(6)}$.The pulse repetition rate is $0.83 \mathrm{~Hz}$, i.e. one pulse every $1.2 \mathrm{~s}$.

A detailed description of the causes of the losses, as well as of the beam cycles that generate them can be found in ref. (7). The measurements were first performed in the three locations described above.
A systematic intercomparison was then carried out in controlled conditions with six detectors at the beginning of the access tunnel to SS16.

\section{Measurements in the three locations}

The measurements were performed by installing the detectors in the three locations and by integrating the number of counts for a period varying between 0.5 and $6 \mathrm{~h}$, according to the different $H^{*}(10)$ rate. The calibration factors applied to the counts were obtained in the CERN calibration laboratory with a PuBe source. The results of the measurements are shown in Table 1 with the statistical uncertainties. The uncertainty on the calibration ( $7 \%$, as reported in the calibration certificate of the source) was not taken into account since it is a correlated uncertainty. For location 3 (access tunnel to SS16) the results of the first series of measurements are not shown since they were quite contradictory due to the high $H^{*}(10)$ rate gradient in the area. The measurements were then repeated and the results of the systematic intercomparison are given later in the paper.

\section{Intercomparison at the access tunnel to SS16}

An intercomparison exercise was organised in at the beginning of the access tunnel to SS16 with six 


\section{E. AZA ET AL.}

detectors. They were installed in six reference positions (1-6), and progressively interchanged to obtain a comparison of the responses in all the positions. The tunnel width is $\sim 4 \mathrm{~m}$, while each position is $50 \mathrm{~cm}$ far from the adjacent one. Each measurement lasted $30 \mathrm{~min}$ and the attention focused on the integrated number of counts. The calibration factors applied were calculated by folding, when possible, the response function of the detector with the expected neutron spectrum in the area as obtained via FLUKA ${ }^{(8,9)}$ Monte Carlo simulations ${ }^{(10)}$ (see Figure 2).

Otherwise, the calibration factor obtained in the CERN calibration laboratory with a PuBe source was used. To normalise the results obtained in the measurements, expressed in integrated $H^{*}(10)$, two sources were employed: (1) the integrated proton fluence in the PS, as derived from TIMBER ${ }^{(11)}$, a Java interface that allows obtaining data on the operation of the CERN accelerators in terms of setting, particle fluence and beam intensity; (2) the data, expressed in integrated $H^{*}(10)$, recorded by a RAMSES ${ }^{(1)}$ station present in the area. The second source of normalisation is useful in the case of non-constant beam

Table 1. Results of the measurements expressed as integrated $H^{*}(10)$ in $\mathrm{nSv}$, with uncertainties in parenthesis.

\begin{tabular}{llll}
\multicolumn{3}{l}{ Route Goward (beam injection) } \\
LINUS & LUPIN ${ }^{3} \mathrm{He}$ & 2202D & \\
$2310(46)$ & $2385(26)$ & $1465(38)$ & \\
LINAC 3 (beam extraction) & & \\
LINUS & Wendi-2 & LUPIN $^{3} \mathrm{He}$ & LB6411 \\
$322(17)$ & $327(18)$ & $326(9)$ & $240(13)$ \\
\hline
\end{tabular}

losses during the measurements; otherwise it can be used as cross-check normalisation. Tables 2 and 3 show the normalised results. The uncertainties are given as sum of two components: the statistical one and the positioning one $(5 \%)$.

Figures 3 and 4 show the plot of the results as normalised with both methods. When not visible, the uncertainty bars are smaller than the marker size.

\section{DISCUSSION}

\section{Measurements in the three locations}

Table 1 shows that in location 1 (Route Goward) the results of the extended range rem counters, i.e. LINUS and LUPIN ${ }^{3} \mathrm{He}$, are consistent within their uncertainties, while 2202D measures $\sim 40 \%$ less. As expected, due to the relatively long and smoothed time structure of the beam losses, the results are not affected by dead-time losses. This is confirmed by the fact that LUPIN ${ }^{3} \mathrm{He}$ and LINUS, which work with different electronics, measure approximately the same $H^{*}(10)$ value. The underestimation of $2202 \mathrm{D}$ can be explained by its low sensitivity for neutron energies $>20 \mathrm{MeV}$, while the expected neutron spectrum in the area is characterised by a peak at $80 \mathrm{MeV}$, due to the primary spallation process ${ }^{(7)}$.

In location 2 (LINAC 3 building) the two categories of instruments (conventional and extended range rem counters) show different readings, as expected from their response functions. LINUS, Wendi-2 and LUPIN ${ }^{3} \mathrm{He}$ measure similar $H^{*}(10)$ values, which are coherent with the value obtained via FLUKA simulations, while LB6411 underestimates by $30 \%$. This is due to the expected neutron spectrum in the

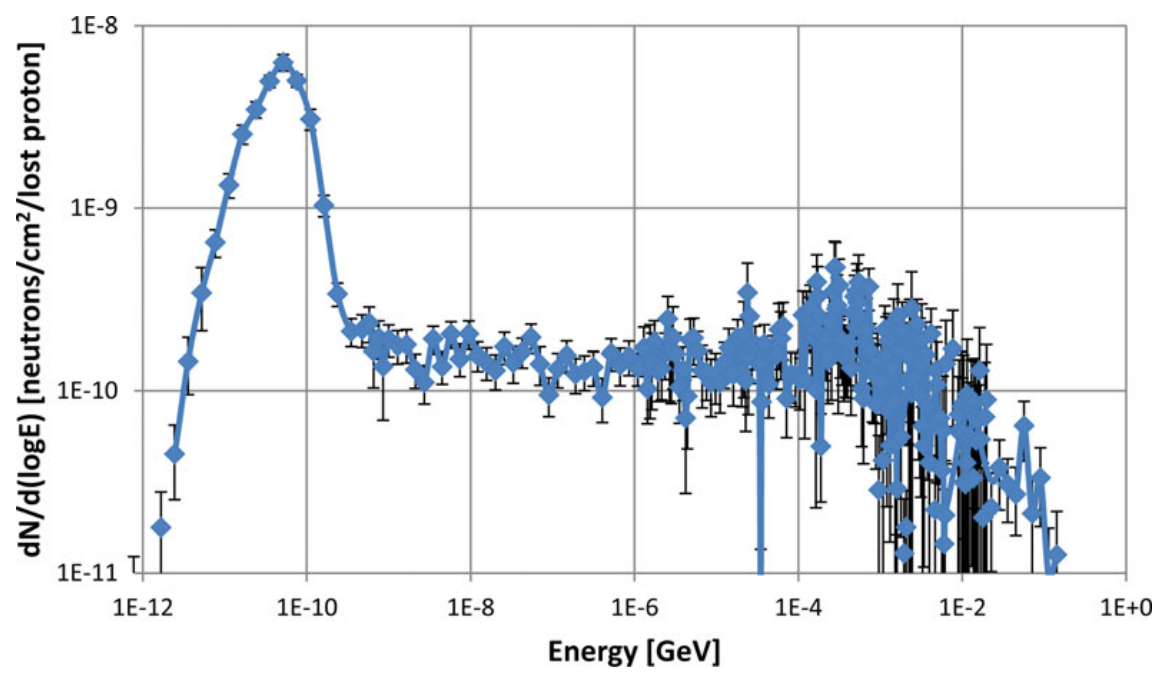

Figure 2. The plot of the neutron spectrum expected at the access tunnel to SS16 as obtained via FLUKA simulations. 
INSTRUMENT INTERCOMPARISON AROUND THE CERN PS

Table 2. Results of the intercomparison measurements, as normalised to the $H^{*}(10)$ integrated by the $\operatorname{RAMSES}^{(1)}$ station, expressed as absolute ratios, with uncertainties in parenthesis.

\begin{tabular}{lrccrrr}
\hline Detector & Pos. 1 & Pos. 2 & Pos. 3 & Pos. 4 & Pos. 5 & Pos. 6 \\
\hline LINUS & $36.0(2.7)$ & $39.7(3.0)$ & $40.6(3.1)$ & $36.0(3.4)$ & $41.6(3.1)$ & $38.1(3.3)$ \\
LUPIN BF & $104.9(7.1)$ & $134.2(8.4)$ & $149.1(8.2)$ & $145.9(9.2)$ & $139.5(9.4)$ & $135.7(8.4)$ \\
H chamber & $90.7(6.1)$ & $122.3(8.3)$ & $125.3(8.8)$ & $128.0(9.0)$ & $128.3(8.3)$ & $123.3(8.4)$ \\
Ar chamber & $97.3(5.0)$ & $130.6(6.2)$ & $147.8(6.4)$ & $160.1(6.2)$ & $154.4(6.1)$ & $157.1(5.8)$ \\
BIOREM & $67.2(9.2)$ & $90.3(11.7)$ & $89.8(12.4)$ & $86.4(13.1)$ & $86.6(12.7)$ & $83.7(12.5)$ \\
Wendi-2 & $40.3(2.7)$ & $45.6(3.3)$ & $45.0(3.4)$ & $47.8(3.4)$ & $46.8(3.3)$ & $46.0(3.0)$ \\
\hline
\end{tabular}

Table 3. Results of the intercomparison measurements, as normalised to the integrated proton fluence in the PS, expressed in $\mathrm{nSv}$ per $10^{13}$ protons, with uncertainties in parenthesis.

\begin{tabular}{lrrrrrr}
\hline Detector & Pos. 1 & Pos. 2 & Pos. 3 & Pos. 4 & Pos. 5 & Pos. 6 \\
\hline LINUS & $9.4(0.6)$ & $11.6(0.7)$ & $10.6(0.7)$ & $12.4(0.8)$ & $10.4(0.7)$ & $10.9(0.7)$ \\
LUPIN BF & $26.3(1.5)$ & $34.7(1.9)$ & $37.0(2.1)$ & $36.5(2.0)$ & $39.6(2.2)$ & $31.9(1.8)$ \\
H chamber & $21.7(1.3)$ & $29.3(1.7)$ & $34.8(2.0)$ & $38.6(2.2)$ & $31.2(1.8)$ & $33.1(1.9)$ \\
Ar chamber & $25.8(1.2)$ & $36.3(1.3)$ & $36.2(1.3)$ & $37.2(1.4)$ & $40.2(1.5)$ & $43.4(1.3)$ \\
BIOREM & $19.8(2.0)$ & $23.0(2.7)$ & $23.2(2.6)$ & $24.8(2.7)$ & $26.8(2.9)$ & $22.0(3.1)$ \\
Wendi-2 & $11.6(0.7)$ & $12.6(0.7)$ & $13.5(0.8)$ & $12.5(0.7)$ & $12.0(0.7)$ & $11.9(0.7)$ \\
\hline
\end{tabular}

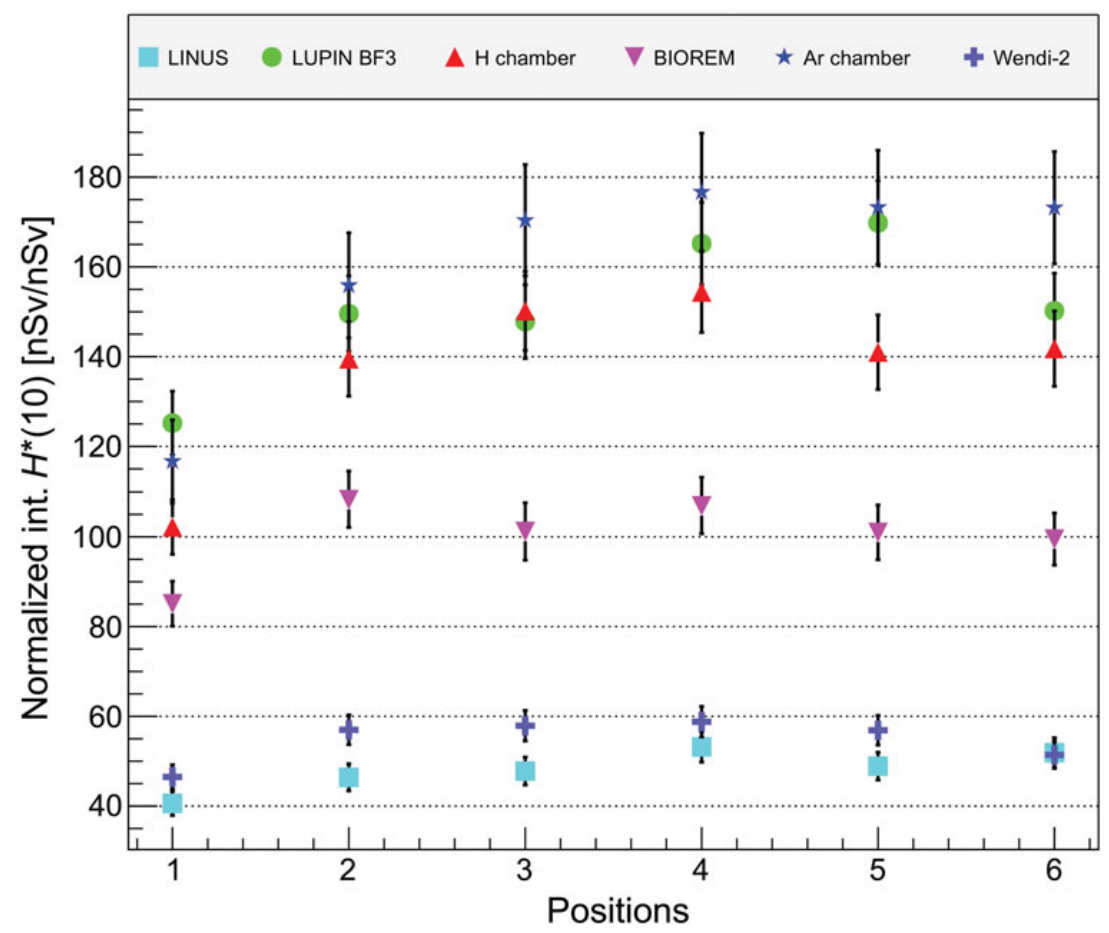

Figure 3. Results of the intercomparison, as normalised to the $H^{*}(10)$ integrated by the RAMSES ${ }^{(1)}$ station, expressed as absolute ratios. 
E. AZA ETAL.

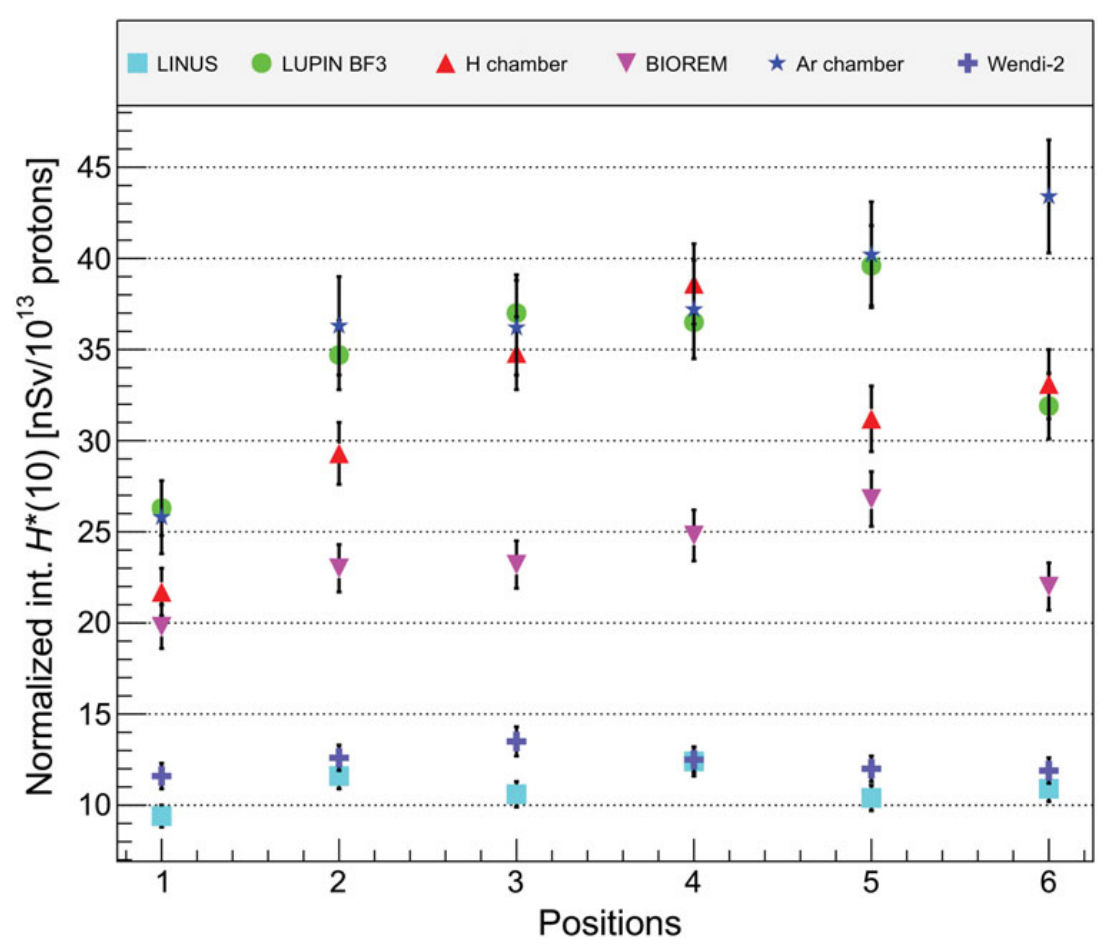

Figure 4. Results of the intercomparison, as normalised to the integrated proton fluence in the PS, expressed in nSv per $10^{13}$ protons.

area, whose part $>20 \mathrm{MeV}$ is of some importance. It can be assumed that the acquisitions were not affected by dead-time losses due to the low $H^{*}(10)$ rate in the area that did not produce pile up effects in the detectors.

\section{Intercomparison at the access tunnel to SS16}

From Figures 3 and 4 the detectors employed in the measurements can be divided in three classes: (1) the two ionisation chambers and LUPIN $\mathrm{BF}_{3}$; (2) BIOREM and (3) LINUS and Wendi-2. The reading of the first three detectors agree well amongst them within the range of uncertainty and are coherent with what expected from FLUKA simulations (i.e. $20-40 \mathrm{nSv}$ per $10^{13}$ protons); the BIOREM underestimates by $30 \%$; the last class of detectors underestimates by $65 \%$. This is explained by dead-time losses, which are higher in the last classes of detectors, characterised by a dead-time of $\sim 2 \mu \mathrm{s}$, while the BIOREM has a dead-time of $\sim 1 \mu \mathrm{s}$.

It can also be noticed that there is a slight difference in the measured $H^{*}(10)$ values between the six reference positions, which is a confirmation of the high $H^{*}(10)$ rate gradient in the area. From the comparison between the two plots one can also see that the data normalised to the $H^{*}(10)$ integrated by the RAMSES $^{(1)}$ station are much more stable than the data normalised to the PS proton fluence. This is probably due to the fact that the fraction of lost beam did not stay constant during the measurements.

\section{CONCLUSIONS}

The analysis of the results obtained in the three locations and during the intercomparison at the access tunnel to SS16 shows that:

(1) conventional rem counters (Berthold LB6411 and Studsvik 2202D) underestimate $H^{*}(10)$ by $\sim 30 \%$ with respect to the extended range rem counters and the FLUKA expected value, due to their low sensitivity for neutron energies $>20 \mathrm{MeV}$, when exposed in locations where the beam losses in the PS produce high-energy stray fields;

(2) extended range rem counters (LUPIN, LINUS and Wendi-2) agree well amongst them and with the FLUKA expected $H^{*}(10)$ value when exposed in a non-pulsed high-energy stray field;

(3) all the rem counters apart from the LUPIN (LINUS, Wendi-2, BIOREM) showed important dead-time losses when exposed to pulsed neutron stray fields that lead to a consistent underestimation 


\section{INSTRUMENT INTERCOMPARISON AROUND THE CERN PS}

of $H^{*}(10)$, from $30 \%$ up to $65 \%$. This is due to dead-time losses that are higher in the detectors characterised by a higher dead-time;

(4) ionisation chambers readings agree with the range of uncertainty and with the FLUKA predictions. These detectors are not affected by deadtime losses and their reliability is high even when employed in an intense pulsed neutron field;

(5) LUPIN showed results coherent with the $H^{*}(10)$ values as obtained via FLUKA simulations and did not show underestimation of $H^{*}(10)$ in all the measurement locations, proving its ability to efficiently withstand very intense pulsed fields.

\section{ACKNOWLEDGEMENTS}

The authors wish to thank Gerald Dumont and Vincent Donate for their support in the experimental activity.

\section{FUNDING}

This work has been supported by a Marie Curie Early Initial Training Network Fellowship of the European Community's Seventh Framework Programme under contract number PITN-GA-2011-289198-ARDENT.

\section{REFERENCES}

1. Segura Millan, G., Perrin, D. and Scibile, L. RAMSES: the LHC radiation monitoring system for the environment and safety. In: Proceedings of the 10th International Conference on Accelerator \& Large Experimental Physics Control Systems, Geneva, Switzerland, 10-14 October 2005, TH3B.1-3O (2006).

2. Birattari, C., Esposito, A., Ferrari, A., Pelliccioni, M., Rancati, T. and Silari, M. The extended range neutron rem counter 'LINUS': overview and latest developments. Radiat. Prot. Dosim. 76, 135-148 (1998).

3. Caresana, M., Ferrarini, M., Manessi, G. P., Silari, M. and Varoli, V. LUPIN: a new instrument for pulsed neutron fields. Nucl. Instrum. Methods A 712, 15-26 (2013).

4. Aza, E., Caresana, M., Cassell, C., Charitonidis, N., Harrouch, E., Manessi, G. P., Pangallo, M., Perrin, D., Samara, E. and Silari, M. Instrument intercomparison in the pulsed neutron fields at the CERN HiRadMat facility. CERN Technical Note, CERN-RP-2013-037REPORTS-TN. CERN.

5. Caresana, M., Helmecke, M., Kubancak, J., Manessi, G. P., Ott, K., Scherpelz, R. and Silari, M. Instrument intercomparison in the high energy mixed field at the $C E R N-E U$ reference field (CERF) facility. In: Proceedings of the 12th NEUtron and ion DOSimetry symposium (NEUDOS), 3-7 June, Aix-en-Provence, France. Radiation Protection Dosimetry (2013).

6. Barranco, J. and Gilardoni, S. Simulation and optimization of beam losses during continuous transfer extraction at the CERN proton synchrotron. Physical Review Special Topics - Accelerators and Beams 14 (2011) 030101 .

7. Caresana, M., Gilardoni, S., Malacrida, F., Manessi, G. P. and Silari, M. Environmental measurements and instrument intercomparison around the PS accelerator complex. CERN Technical Note. CERN-DGS-2012036-RP-TN (2012).

8. Battistoni, G., Muraro, S., Sala, P. R., Cerutti, F., Ferrari, A., Roesler, S., Fasso, A. and Ranft, J. The FLUKA code: description and benchmarking. In: Proceedings of the Hadronic Shower Simulation Workshop 2006, Fermilab, USA, 6-8 September 2006. Albrow M. and Raja R. Eds., AIP Conference Proceeding 896, pp. 31-49 (2007).

9. Ferrari, A., Sala, P. R., Fasso, A. and Ranft, J. FLUKA: a multi-particle transport code. CERN Technical Note, CERN-2005-10 (2005). INFN/TC-05/11, SLAC-R-773. CERN.

10. Damjanovic, S., Otto, T. and Widorski, M. Shielding improvements in the region of the ejection septum SS16 of the CERN PS. CERN Technical Note, CERN-SC2010-022-RP-TN. CERN.

11. Billen, R. and Roderick, C. The LHC logging service: capturing, storing and using time-series data for the world's largest scientific instrument. CERN Technical Note, CERN-AB-Note-2006-046 (2006 ). CERN. 